\title{
Pulmonary rehabilitation in patients with an acute exacerbation of chronic obstructive pulmonary disease
}

\author{
Sarah E. Jones ${ }^{1,2 \#}$, Ruth E. Barker ${ }^{1 \#}$, Claire M. Nolan ${ }^{1,2}$, Suhani Patel ${ }^{1}$, Matthew Maddocks ${ }^{3}$, William D. C. \\ $\operatorname{Man}^{1,2}$ \\ ${ }^{1}$ Harefield Pulmonary Rehabilitation and Muscle Research Laboratory, Royal Brompton and Harefield NHS Foundation Trust, UK; ${ }^{2}$ National Heart \\ and Lung Institute, Imperial College, London, UK; ${ }^{3}$ King's College London, Cicely Saunders Institute of Palliative Care, Policy and Rehabilitation, \\ London, UK \\ Contributions: (I) Conception and design: S Jones, R Barker, M Maddocks, W Man; (II) Administrative support: None; (III) Provision of study \\ materials or patients: None; (IV) Collection and assembly of data: S Jones, R Barker; (V) Data analysis and interpretation: None; (VI) Manuscript \\ writing: All authors; (VII) Final approval of manuscript: All authors. \\ \#These authors contributed equally to this work. \\ Correspondence to: Sarah E. Jones. Harefield Pulmonary Rehabilitation and Muscle Research Laboratory, Harefield Hospital, Hill End Road, Harefield \\ UB9 6JH, UK. Email: s.jones5@rbht.nhs.uk; Ruth E. Barker. Harefield Pulmonary Rehabilitation and Muscle Research Laboratory, Harefield \\ Hospital, Hill End Road, Harefield UB9 6JH, UK. Email: r.barker2@rbht.nhs.uk.
}

\begin{abstract}
Acute exacerbations of chronic obstructive pulmonary disease (AECOPD) are one of the most common causes of emergency hospital admission and place great burden upon healthcare systems. Furthermore, AECOPD represent an important life event for patients, and are associated with significant reductions in physical activity, skeletal muscle function, exercise tolerance and health-related quality of life. Pulmonary rehabilitation, an intervention comprising supervised exercise-training and education, may counteract these negative consequences and target modifiable risk factors for hospital readmission. A recent Cochrane systematic review included 20 randomized controlled trials comparing pulmonary rehabilitation after exacerbation of COPD versus conventional care. Overall, the evidence supports moderate to large effects on health-related quality of life and exercise capacity. However, there is substantial heterogeneity across studies, and more recent studies have been more equivocal, including around hospital readmissions, particularly when rehabilitation is started in the inpatient setting. In this narrative review, we examine the rationale for pulmonary rehabilitation following AECOPD with a particular focus on skeletal muscle function, review the current evidence for pulmonary rehabilitation in the AECOPD setting, and identify areas that require future research, including the structure and nature of the intervention, improving uptake and adherence, and the role of alternative rehabilitation strategies for patients with AECOPD.
\end{abstract}

Keywords: Acute exacerbation; chronic obstructive pulmonary disease; exercise; rehabilitation; skeletal muscle dysfunction

Submitted Feb 22, 2018. Accepted for publication Feb 28, 2018.

doi: $10.21037 /$ jtd.2018.03.18

View this article at: http://dx.doi.org/10.21037/jtd.2018.03.18

\section{Introduction}

Acute exacerbations of chronic obstructive pulmonary disease (AECOPD) are one of the commonest causes of emergency hospital admissions and contribute a significant proportion to total COPD healthcare costs (1). In the
United States, inpatient care for COPD was estimated to cost $\$ 11.9$ billion annually (2). Not only are AECOPD costly to healthcare systems, they are associated with poor prognosis. In the United Kingdom, a recent national audit revealed that $43 \%$ of patients hospitalized with AECOPD were readmitted and $12 \%$ died within 90 days (3). From a 
patient perspective, an AECOPD requiring hospitalization is associated with a significant decline in physical activity levels (4), functional capacity (5), skeletal muscle function (6) and health-related quality of life (7).

Pulmonary rehabilitation (PR), defined by the American Thoracic Society/European Respiratory Society (ATS/ERS) as a 'comprehensive intervention based on a thorough patient assessment followed by patient tailored therapies that include, but are not limited to, exercise training, education, and behaviour change, designed to improve the physical and psychological condition of people with chronic respiratory disease' (8), is a cornerstone in the non-pharmacological management of COPD. The evidence base supporting the benefits of PR in stable patients with COPD is substantial, with 65 randomized controlled trials contributing to the most recent Cochrane systematic review (9). In recent years, there has been increasing interest in the role of PR in the acute setting (either during or shortly after a hospital admission for AECOPD). The latest iteration of the Cochrane systematic review of PR in the peri-exacerbation period identified 20 randomized controlled trials and found that PR improved functional capacity and healthrelated quality of life (10). However there was significant heterogeneity in the data, with particularly uncertainty about whether PR in the acute setting reduced rates of hospital readmission. Furthermore, recent trials have shown less marked benefits compared to older trials (10), particularly when rehabilitation is started as an inpatient (11). Despite being recommended in national and international guidelines, there may be problems with the clinical implementation of PR in the AECOPD setting as observational data suggest barriers to referral, uptake and adherence (12).

This narrative review will examine the consequences of an AECOPD, and in particular, we will highlight the effect of AECOPD on skeletal muscle function (in line with the theme of this review series) and other factors that are potentially modifiable by PR. We will summarize the current literature on the effects of PR during/after AECOPD, and finally identify and discuss areas of uncertainty that would benefit from further research.

\section{Consequences of AECOPD}

With AECOPD, there is typically an increase in symptom severity from the baseline state (worsening cough, shortness of breath, sputum production, fatigue) rather than the onset of new symptoms $(13,14)$. These quantitative changes in symptoms have a profound effect upon the patient's physical activity levels. Forty-five percent of patients become bed/couch bound and $55 \%$ have to stop work during a community-treated AECOPD (13). In an interviewbased survey, $86 \%$ of patients reported that an AECOPD had a significant impact on activities of daily living, with $47 \%$ stopping all activities completely (15). This patientreported decline in physical activity levels is corroborated by objective measures using accelerometers. Pitta et al. (4) found that patients hospitalized with an AECOPD spent only $7 \%$ of their time on weight-bearing activities on the second day of hospital admission, and only increasing a further $2 \%$ by hospital discharge. Recent data suggest that moderate AECOPD, treated in the outpatient setting, are also associated with a substantial decrease in daily physical activity levels (16).

Disuse atrophy, secondary to reduced physical activity, is likely to be a significant contributor to skeletal muscle dysfunction during an AECOPD. Studies, where healthy elderly individuals have been consigned to experimental bed rest, have shown large reductions in leg lean mass, muscle strength and muscle fractional synthetic rate (17), which are more pronounced than in younger healthy individuals (18). In COPD, quadriceps force and cross-sectional area decreases by $5 \%$ over five days of hospitalization $(4,6)$ and show only partial recovery by three months after hospitalization (6). Moreover, it is important to remember that a significant proportion of these patients may have co-existing skeletal muscle dysfunction prior to the severe AECOPD (19,20).

Although disuse atrophy is likely to be the central player in the development of skeletal muscle dysfunction during an AECOPD, other potentially contributing factors include systemic inflammation, oxidative stress, blood gas abnormalities, comorbidities, corticosteroids, increased resting metabolic rate and suppressed appetite (Figure 1). These have been reviewed in detail elsewhere $(21,22)$.

\section{Prognostic importance of skeletal muscle dysfunction in AECOPD}

Many consequences of an AECOPD are potentially modifiable risk factors for hospital readmission, including reduced exercise tolerance, physical inactivity, dependency with activities of daily living, and health status $(23,24)$. Recent data also support the prognostic importance of skeletal muscle dysfunction. Greening and colleagues 


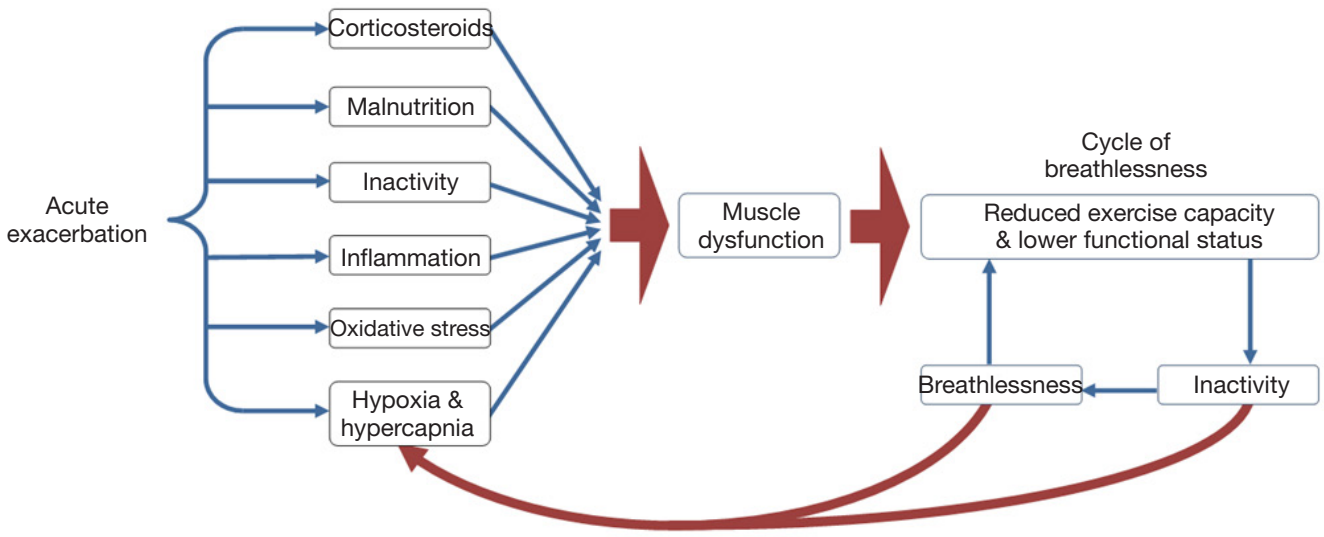

Figure 1 Schematic of the impact of acute exacerbations of COPD upon the cycle of breathlessness (adapted from Burtin et al., 2011).

studied a subgroup of 191 patients participating in a randomized controlled of an early rehabilitation intervention initiated during hospitalization for AECOPD. Those in the lowest quartile for ultrasound-measured rectus femoris cross sectional area (corrected for height squared) spent more days in hospital in the year after index hospitalization than those in the highest quartile [mean (SD) 28.1 (33.9) vs. 12.2 (23.50) days; $\mathrm{P}=0.007)(25)$, and in multivariate analysis, rectus femoris cross sectional area remained an independent risk factor for unscheduled readmission or death [odds ratio (OR) 0.46; 95\% CI, 0.22-0.95; $\mathrm{P}=0.035]$ (25). Kon et al. evaluated the prognostic ability of the four-metre gait speed (4MGS) measured at hospital discharge in patients with AECOPD (26). The 4MGS is a simple measure of lower limb physical performance, and is included in operational definitions of sarcopenia and frailty $(19,27)$. Increased rates of all-cause hospital readmission at 90 days were observed across quartiles of decreasing $4 \mathrm{MGS}$ (fastest: $11.5 \%$; slowest: $48.2 \% ; \mathrm{P}<0.001)$. Compared with those in the fastest 4MGS quartile, those in the slowest quartile had unadjusted ORs (95\% CIs) for 90-day readmission of 7.12 (2.61 to 19.44) (26).

\section{Evidence base for PR in AECOPD}

The impact of PR following an acute exacerbation of COPD was recently updated in a Cochrane systematic review (10). Puhan and colleagues included randomized controlled trials comparing PR with usual care after AECOPD, and incorporated a further 11 studies to a previous iteration of the systematic review to include a total of 20 trials (1,477 participants). PR, which could be delivered in the inpatient and/or outpatient settings, had to commence immediately after initiation of exacerbation treatment or within three weeks of initiation of exacerbation treatment. Overall, PR after an AECOPD appeared to be safe with no increased mortality signal. There was moderate quality evidence supporting a reduction in hospital readmission rates with $\mathrm{PR}$ (pooled OR 0.44, 95\% CI, 0.21 to $0.91, \mathrm{P}=0.03$ ), although results were heterogeneous. The authors suggested that the heterogeneity was explained to some extent by the "extensiveness" of the rehabilitation intervention (using guidelines from international societies $(8,28)$, the authors graded interventions according to the total number, frequency, supervision and content of exercise training sessions, and whether the intervention included a self-management/education programme) as well as by the methodological quality of the included trials. There was high-quality evidence to support improved health-related quality of life and exercise capacity with PR (10), although newer studies appeared to show less marked benefits than seen in earlier trials, or even no benefit at all.

This was best demonstrated by the trial from Greening and colleagues (11) - the study that contributed the most number of patients to the latest Cochrane review. The authors randomized 389 hospitalized patients with COPD to either usual care or a 6-week rehabilitation intervention comprising an inpatient component starting within 48 hours of hospitalization, followed by a post-discharge home-based supported self-management programme facilitated by a manual introduced using motivational interviewing (11). The daily inpatient programme used a range of nonvolitional (neuromuscular electrical stimulation) and volitional (walking, sit-to-stand, inner range contraction against gravity, progressive upper and lower limb muscle 
resistance training) modalities to achieve the highest individualized tolerable intensity of exercise training. The exercise component of the home-based supported selfmanagement programme consisted primarily of progressive walking through goal setting. Greening et al. observed no difference in readmission risk [hazard ratio (HR) 1.10, $95 \% \mathrm{CI}, 0.86$ to $1.43, \mathrm{P}=0.44]$ with intervention, nor any difference in recovery of physical function and health status between intervention and control groups (11).

There were unique aspects to this trial. Strikingly, this is the only trial included within the Cochrane review that has shown a higher risk of death associated with rehabilitation at one year (OR 1.74, 95\% CI, 1.05 to $2.88, \mathrm{P}=0.03$ ). The risk between intervention and control groups did not start diverging until seven months-it seems unlikely that the higher mortality could be directly attributable to the intervention; more likely there was a subtle imbalance of baseline characteristics between the groups (29). Another unique observation was the rate of natural recovery in physical functioning and health status in the usual care group, perhaps reflecting the current trend for early mobilization as part of routine care, in contrast to clinical practice two decades ago when the first PR trials in the acute setting were conducted. Regardless, there seemed to be an insufficient difference in exercise stimulus between the intervention and control groups. The inpatient stay was relatively short (median 5 days) so taking into account time needed to approach and consent, the opportunity to provide supervised exercise training was restricted (less than 3 sessions each of aerobic and resistance training). Adherence to the home self-management exercise programme was poor and there was limited uptake of conventional outpatient $\mathrm{PR}$ in the intervention group compared to usual care (14\% vs. $22 \%, \mathrm{P}=0.04)$ (11). These data, taken together with the findings of the Cochrane review, suggest that intensity, frequency and duration of the exercise component of the intervention are highly relevant.

\section{Skeletal muscle function following PR for an AECOPD}

Despite the prognostic significance of skeletal muscle dysfunction and the inclusion of muscle resistance training in many PR interventions, most trials have focused on health status and exercise capacity outcomes rather than muscle function. The available data on the effects of PR on muscle function is primarily described in studies where rehabilitation takes place during the hospitalization period where resistance training appears to mitigate the loss of muscle strength observed during an admission.

Troosters et al. demonstrated that daily quadriceps resistance training for 7 days on a knee extension chair resulted in a $10 \%$ improvement in quadriceps force (compared with $-1 \%$ in control group) (30), whilst another study showed that an average of 5.6 sessions of whole body resistance training started on third day of admission maintained knee extensor isometric force at discharge in comparison to $>10 \%$ loss of force observed in the control group (31). Frail older patients with COPD appear to particularly benefit from aerobic exercise; a minimum of four sessions of cycling using a simple portable pedal exerciser resulted in significant improvements in lower limb strength, sit to stand, and balance measures compared with usual care (32).

Data in the post-discharge setting is limited. Seymour and colleagues showed that outpatient PR delivered twice a week for eight weeks resulted in a large improvement in quadriceps maximum voluntary contraction $(+5.1 \mathrm{~kg})$ compared with usual care, and this improvement in quadriceps strength was correlated with change in walking exercise capacity (33). Greening et al. showed a progressive increase in quadriceps strength over the 12 months following hospital discharge in both intervention and control groups, intimating that a degree of natural recovery occurs (11). As discussed previously, the exercise stimulus of the intervention was probably insufficient to influence recovery rates of muscle function.

\section{Challenges in delivering PR following an AECOPD}

Despite the evidence-base, as well as universal inclusion of PR in management guidelines for COPD (34), there is global underutilization of PR both in stable outpatients and patients hospitalized with AECOPD $(35,36)$. Recent data have shown there are multiple barriers at all levels of the post-AECOPD PR patient pathway. Jones et al. reported that out of 448 hospital discharges for AECOPD over a 12 -month period, only 90 referrals were made for PR with 43 patients receiving and completing PR (9.6\% of all hospital discharges, $15 \%$ of eligible patients) (Figure 2) (12). Similarly, Harrison et al. found that only 71 of 128 patients hospitalized with AECOPD accepted a referral for PR, of whom only 39 attended initial assessment and 11 finally completing (37).

The reasons for poor referral, uptake and completion rates are complex and can be broadly divided into those 


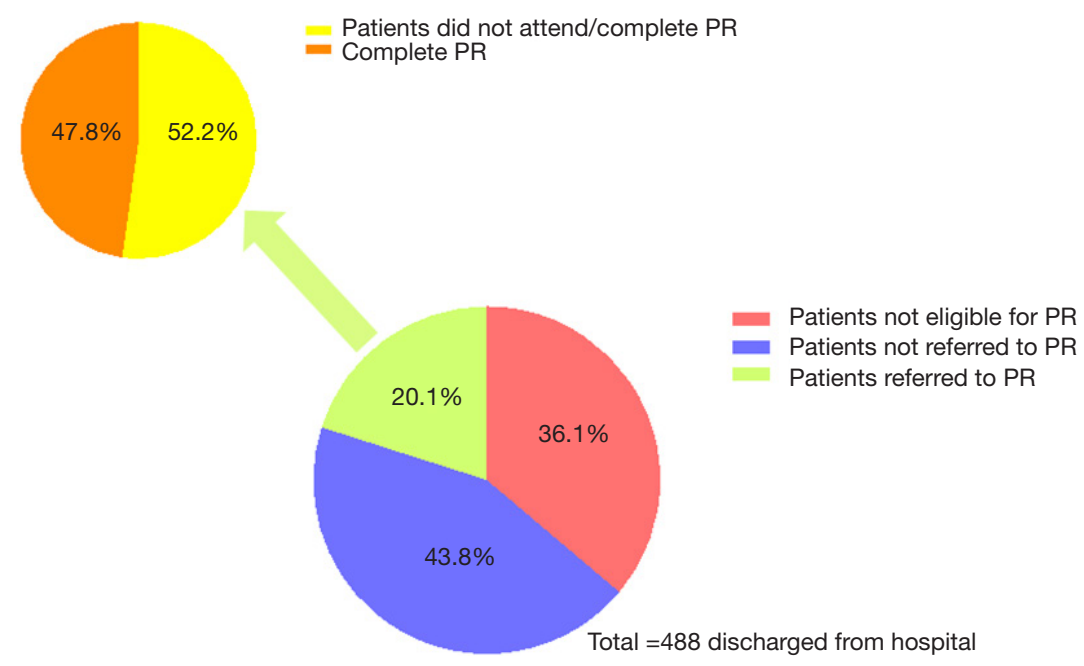

Figure 2 Referral, uptake and adherence data to post-exacerbation pulmonary rehabilitation following an admission to hospital over 12-month in a north-west London hospital, UK (adapted from Jones et al., 2013).

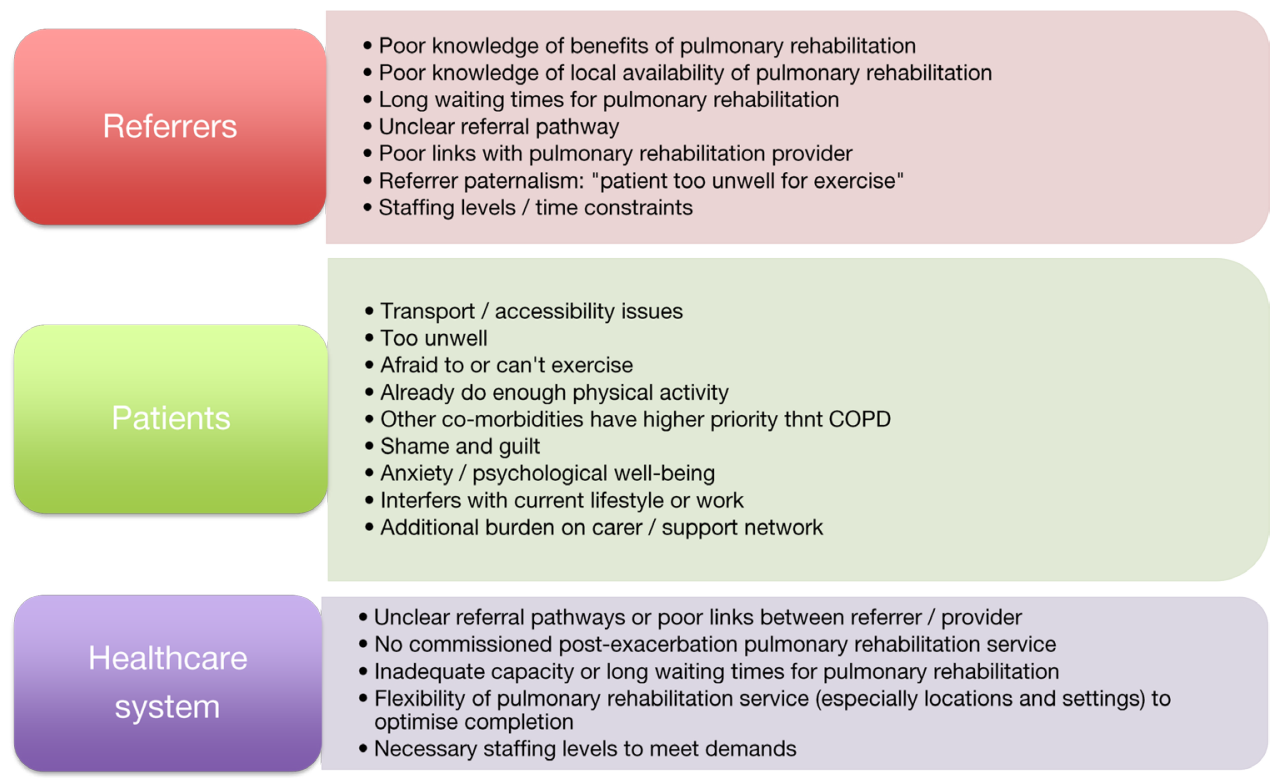

Figure 3 Reasons for poor referral, uptake and completion rates to post-exacerbation pulmonary rehabilitation.

related to healthcare systems, referrers and patients (Figure 3). The gap between trial evidence to clinical implementation may in part by explained by issues within healthcare systems such as insufficient resources or reimbursement that limit the provision of PR. Previous audit data from the United Kingdom showed that a minority of established services were able to offer PR within four weeks of hospital discharge, and data from post-
AECOPD PR contributed less than $2 \%$ of the total audit dataset (38). Other healthcare system considerations include a lack of integration between inpatient teams managing the hospital admission and outpatient PR teams. There are no data examining referrer attitudes to post-AECOPD PR, but existing data show significant dropouts at the referral stage (12) (Figure 2). This could reflect lack of referrer awareness/education regarding PR, time constraints, 
paternalistic attitudes or simply a result of patient concerns/ desires.

From a patient perspective, the most commonly cited reasons for declining referral include transport issues, feeling too unwell, not being interested in physical activity after an exacerbation, satisfaction with current functional ability and being busy with other activities $(37,39,40)$. Patients may justify by describing their own attempts at increasing physical activity ("I do arm exercises and try to walk quickly when I can") and how PR might encroach on their normal daily activities ("I would feel that the time I'm going there I could be doing things that I want to do at home, and I'm happy doing things at home") (41). Previous experience of $\mathrm{PR}$ may also influence whether a patient decides to accept a referral, with intensity of exercise and bad experiences emerging as themes (40). Benzo et al. (39) reported that $11 \%$ of patients declined referral to $P R$ because they felt that there was too much "going on" or were "overwhelmed". Hospital admissions for AECOPD are life-changing events and the timing of PR referral may be highly relevant to some individuals. Even with uptake of $\mathrm{PR}$, patient attitudes towards the rehabilitation intervention may be a barrier to completion. As a sub-study of the previously described trial by Greening and colleagues (11), Vincent et al. (42) reported the perspectives and experiences of participants in the intervention group. At 48 hours following discharge, $9 \%$ of patients reported "feeling unwell", $8 \%$ were "feeling worried", $15 \%$ reported "finding the exercise difficult", whilst only $2 \%$ said that they "felt more active". By the end of the intervention, only $44 \%$ of the patients in this study felt that exercise had had a positive effect on their recovery.

\section{Can uptake and completion of PR be improved?}

Limited data exist regarding interventions to increase uptake and completion of PR. A recent systematic review (43) was only able to identify one quasi-randomised control study that examined whether the allocation of a tablet computer could improve completion of pulmonary rehabilitation in patients with stable COPD (44), and was unable to identify any completed trials that aimed to increase uptake or completion of post-AECOPD PR. Service improvement strategies, such as COPD discharge bundles (a structured aide memoire of evidence-based practices), have shown some promise in facilitating the uptake of post-AECOPD PR (45), but are themselves challenging to implement (46).

Transport and travel are commonly cited as barriers to uptake and completion of PR (47), particularly in the outpatient setting. Even with the provision of door-todoor transport, adherence to outpatient-based PR remains suboptimal (48). Home-based PR is therefore potentially attractive, particularly as recent home-based PR trials in patients with stable disease have shown non-inferiority to traditional supervised outpatient-based PR $(49,50)$. However, as the Cochrane review on post-exacerbation PR (10) has informed, less "extensive" and lightly supervised PR interventions may not be effective in highly symptomatic and breathless patients following AECOPD $(11,51,52)$. Supervised home-based PR in the acute setting has been trialled alongside hospital at home schemes, which traditionally consist of home-based, pharmacological management of the AECOPD under nurse-supervision (53). Murphy et al. provided twice-weekly supervised exercise training for 6 weeks alongside a hospital at home service and demonstrated improvements in exercise capacity, muscle strength and quality of life in the intervention, but not control, group (54). However, sample sizes were small and between-group changes were not reported. Larger, more rigorous trials are required to explore the efficacy and costeffectiveness of home-based PR in the post-discharge setting.

Another reported barrier to $\mathrm{PR}$ is patient perception that they are too unwell to partake in aerobic or resistance exercise training. Investigators have explored less effort-dependent rehabilitation strategies, including neuromuscular electrical stimulation (NMES) and whole body vibration (WBV). NMES uses a portable stimulator and skin electrodes to produce a controlled contraction of the muscle, typically the quadriceps. Maddocks et al. demonstrated that a six-week course of quadriceps NMES was sufficient to improve functional capacity, quadriceps strength and ultrasound measured cross-sectional area of the rectus femoris in patients with severe COPD and significant respiratory disability (55). The most recent Cochrane systematic review of 18 randomised control trials comparing NMES with resistance training or no treatment or placebo in patients with advanced disease found that NMES had a statistically significant improvement in quadriceps muscle strength compared to control (standard mean difference $0.53,95 \%$ confidence interval 0.19 to 0.87 ), equating to a difference of $1.1 \mathrm{~kg}$ (56). Pilot studies using NMES in hospitalized patients with AECOPD have shown that it is both feasible and may improve quadriceps strength (57-59). However the largest study to use NMES in patients hospitalized with AECOPD was only able to deliver a mean 3.6 sessions of NMES prior to hospital discharge and 
was unable to influence recovery in physical performance or health status compared with usual care (11). WBV is a training modality performed on a vibrating platform that moves in sinusoidal oscillations, during which static and dynamic exercises can be performed. A recent meta-analysis of studies in patients with COPD showed that WBV improves six minutes walk distance compared to control (57.9 m, 95\% CI 16.4 to $99.3 \mathrm{~m}$ ) (60). Only one small study has examined the effect of WBV in the AECOPD setting but was found to improve exercise capacity and health status without any adverse effects (61). Whether such interventions are cost-effective and can be widely implemented in healthcare systems remain unknown.

\section{Conclusions}

Acute exacerbations requiring hospitalization are an important life event for patients with COPD and have devastating impact on physical activity levels, skeletal muscle function and exercise tolerance. Pulmonary rehabilitation produces moderate to large benefits in health-related quality of life and exercise capacity following AECOPD, with the largest effects seen with more extensive and supervised interventions. However significant barriers to referral, patient uptake and completion exist, and there is an urgent need for further research into increasing and improving accessibility to rehabilitation following AECOPD.

\section{Acknowledgements}

Funding: S Jones is funded by a NIHR Doctoral Research Fellowship (DRF-2015-08-004); R Barker is funded by a NIHR Research for Patient Benefit grant (PB-PG-0213-30003); C Nolan is funded by a NIHR Doctoral Research Fellowship (DRF-2014-07-089); M Maddocks is supported by a NIHR Career Development Fellowship (CDF-2017-10-009), the NIHR Collaboration for Leadership in Applied Health Research and Care for South London and Cicely Saunders International; W Man is part funded by the NIHR CLAHRC for NW London and was funded by a NIHR Clinical Scientist award (CS/7/007), NIHR Clinical Trials Fellowship (NIHRCTF-01-12-04) and Medical Research Council (MRC) New Investigator Grant (G1002113).

\section{Footnote}

Conflicts of Interest: The authors have no conflicts of interest to declare.

\section{References}

1. Toy EL, Gallagher KF, Stanley EL, et al. The economic impact of exacerbations of chronic obstructive pulmonary disease and exacerbation definition: a review. COPD 2010;7:214-28.

2. Perera PN, Armstrong EP, Sherrill DL, et al. Acute exacerbations of COPD in the United States: inpatient burden and predictors of costs and mortality. COPD 2012;9:131-41.

3. Stone RA, Holzhauer-Barrie J, Lowe D, et al. COPD: Who cares when it matters most? National Chronic Obstructive Pulmonary Disease (COPD) Audit Programme: Outcomes from the clinical audit of COPD exacerbations admitted to acute units in England 2014. Results and data analysis. 2017. Available online: https:// www.rcplondon.ac.uk/projects/outputs/copd-who-caresorganisational-audit-2014

4. Pitta F, Troosters T, Probst VS, et al. Physical activity and hospitalization for exacerbation of COPD. Chest 2006;129:536-44.

5. Cote CG, Dordelly LJ, Celli BR. Impact of COPD exacerbations on patient-centered outcomes. Chest 2007;131:696-704.

6. Spruit MA, Gosselink R, Troosters T, et al. Muscle force during an acute exacerbation in hospitalised patients with COPD and its relationship with CXCL8 and IGF-I. Thorax 2003;58:752-6.

7. Schmier JK, Halpern MT, Higashi MK, et al. The quality of life impact of acute exacerbations of chronic bronchitis (AECB): a literature review. Qual Life Res 2005;14:329-47.

8. Spruit MA, Singh SJ, Garvey C, et al. An official American Thoracic Society/European Respiratory Society statement: key concepts and advances in pulmonary rehabilitation. Am J Respir Crit Care Med 2013;188:e13-64.

9. McCarthy B, Casey D, Devane D, et al. Pulmonary rehabilitation for chronic obstructive pulmonary disease. Cochrane Database Syst Rev 2015:CD003793.

10. Puhan MA, Gimeno-Santos E, Cates CJ, et al. Pulmonary rehabilitation following exacerbations of chronic obstructive pulmonary disease. Cochrane Database Syst Rev 2016;12:CD005305.

11. Greening NJ, Williams JE, Hussain SF, et al. An early rehabilitation intervention to enhance recovery during hospital admission for an exacerbation of chronic respiratory disease: randomised controlled trial. BMJ 2014;349:4315. 
12. Jones SE, Green SA, Clark AL, et al. Pulmonary rehabilitation following hospitalisation for acute exacerbation of COPD: referrals, uptake and adherence. Thorax 2014;69:181-2.

13. Miravitlles M, Zalacain R, Murio C, et al. Speed of recovery from acute exacerbations of chronic obstructive pulmonary disease after treatment with antimicrobials : results of a two-year study. Clin Drug Investig 2003;23:439-50.

14. Jones PW, Chen WH, Wilcox TK, et al. Characterizing and quantifying the symptomatic features of COPD exacerbations. Chest 2011;139:1388-94.

15. Kessler R, Stahl E, Vogelmeier C, et al. Patient understanding, detection, and experience of COPD exacerbations: an observational, interview-based study. Chest 2006;130:133-42.

16. Alahmari AK, Almalki ZS, Alahmari AK, et al. Thromboembolic events associated with bevacizumab plus chemotherapy for patients with colorectal cancer: a metaanalysis of randomized controlled trials. Am Health Drug Benefits 2016;9:221-32.

17. Kortebein P, Ferrando A, Lombeida J, et al. Effect of 10 days of bed rest on skeletal muscle in healthy older adults. JAMA 2007;297:1772-4.

18. Tanner RE, Brunker LB, Agergaard J, et al. Age-related differences in lean mass, protein synthesis and skeletal muscle markers of proteolysis after bed rest and exercise rehabilitation. J Physiol 2015;593:4259-73.

19. Jones SE, Maddocks M, Kon SS, et al. Sarcopenia in COPD: prevalence, clinical correlates and response to pulmonary rehabilitation. Thorax 2015;70:213-8.

20. Man WD, Soliman MG, Nikoletou D, et al. Nonvolitional assessment of skeletal muscle strength in patients with chronic obstructive pulmonary disease. Thorax 2003;58:665-9.

21. Burtin C, Decramer M, Gosselink R, et al. Rehabilitation and acute exacerbations. Eur Respir J 2011;38:702-12.

22. Abdulai RM, Jensen TJ, Patel NR, et al. Deterioration of limb muscle function during acute exacerbation of chronic obstructive pulmonary disease. Am J Respir Crit Care Med 2018;197:433-49.

23. Maddocks M, Kon SS, Singh SJ, et al. Rehabilitation following hospitalization in patients with COPD: can it reduce readmissions? Respirology 2015;20:395-404.

24. Steer J, Gibson GJ, Bourke SC. Predicting outcomes following hospitalization for acute exacerbations of COPD. QJM 2010;103:817-29.

25. Greening NJ, Harvey-Dunstan TC, Chaplin EJ, et al. Bedside assessment of quadriceps muscle by ultrasound after admission for acute exacerbations of chronic respiratory disease. Am J Respir Crit Care Med 2015;192:810-6.

26. Kon SS, Jones SE, Schofield SJ, et al. Gait speed and readmission following hospitalisation for acute exacerbations of COPD: a prospective study. Thorax 2015;70:1131-7.

27. Maddocks M, Kon SS, Canavan JL, et al. Physical frailty and pulmonary rehabilitation in COPD: a prospective cohort study. Thorax 2016;71:988-95.

28. Bolton CE, Bevan-Smith EF, Blakey JD, et al. British Thoracic Society guideline on pulmonary rehabilitation in adults. Thorax 2013;68 Suppl 2:ii1-30.

29. Man WD, Kon SS, Maddocks M. Rehabilitation after an exacerbation of chronic respiratory disease. BMJ 2014;349:4370.

30. Troosters T, Probst VS, Crul T, et al. Resistance training prevents deterioration in quadriceps muscle function during acute exacerbations of chronic obstructive pulmonary disease. Am J Respir Crit Care Med 2010;181:1072-7.

31. Borges RC, Carvalho CR. Impact of resistance training in chronic obstructive pulmonary disease patients during periods of acute exacerbation. Arch Phys Med Rehabil 2014;95:1638-45.

32. Torres-Sanchez I, Valenza MC, Cabrera-Martos I, et al. Effects of an exercise intervention in frail older patients with chronic obstructive pulmonary disease hospitalized due to an exacerbation: a randomized controlled trial. COPD 2017;14:37-42.

33. Seymour JM, Moore L, Jolley CJ, et al. Outpatient pulmonary rehabilitation following acute exacerbations of COPD. Thorax 2010;65:423-8.

34. NICE. Chronic obstructive pulmonary disease in adults: Quality statement 5: Pulmonary rehabilitation after an acute exacerbation. 2011. Available online: https://www.nice.org.uk/guidance/qs10/chapter/ quality-statement-5-pulmonary-rehabilitation-afteran-acute-exacerbation

35. Rochester CL, Vogiatzis I, Holland AE, et al. An Official American Thoracic Society/European Respiratory Society Policy Statement: enhancing implementation, use, and delivery of pulmonary rehabilitation. Am J Respir Crit Care Med 2015;192:1373-86.

36. Moore E, Newson R, Joshi M, et al. Effects of pulmonary rehabilitation on exacerbation number and severity in people with COPD: an historical cohort study using 
electronic health records. Chest 2017;152:1188-202.

37. Harrison SL, Robertson N, Graham CD, et al. Can we identify patients with different illness schema following an acute exacerbation of COPD: a cluster analysis. Respir Med 2014;108:319-28.

38. Steiner MC, Roberts CM. Pulmonary rehabilitation: the next steps. Lancet Respir Med 2016;4:172-3.

39. Benzo R, Wetzstein M, Neuenfeldt P, et al. Implementation of physical activity programs after COPD hospitalizations: Lessons from a randomized study. Chron Respir Dis 2015;12:5-10.

40. Thorpe O, Kumar S, Johnston K. Barriers to and enablers of physical activity in patients with COPD following a hospital admission: a qualitative study. Int J Chron Obstruct Pulmon Dis 2014;9:115-28.

41. Harrison SL, Robertson N, Apps L, et al. "We are not worthy"--understanding why patients decline pulmonary rehabilitation following an acute exacerbation of COPD. Disabil Rehabil 2015;37:750-6.

42. Vincent EE, Chaplin EJ, Williams JE, et al. Experiences of patients undergoing pulmonary rehabilitation during an exacerbation of chronic respiratory disease. Chron Respir Dis 2017;14:298-308.

43. Jones AW, Taylor A, Gowler H, et al. Systematic review of interventions to improve patient uptake and completion of pulmonary rehabilitation in COPD. ERJ Open Res 2017;3:00089-2016.

44. Ringbaek TJ, Lavesen M, Lange P. Tablet computers to support outpatient pulmonary rehabilitation in patients with COPD. Eur Clin Respir J 2016;3:31016.

45. Sewell L, Schreder S, Steiner M, et al. A strategy to implement a chronic obstructive pulmonary disease discharge care bundle on a large scale. Future Hosp J 2017;4:198-201.

46. Man WD-C, Barker R, Maddocks M, et al. Outcomes from hospitalised acute exacerbations of COPD: a bundle of optimism? Thorax 2017;72:8-9.

47. Keating A, Lee A, Holland AE. What prevents people with chronic obstructive pulmonary disease from attending pulmonary rehabilitation? A systematic review. Chron Respir Dis 2011;8:89-99.

48. 48. Eaton T, Young P, Fergusson W, et al. Does early pulmonary rehabilitation reduce acute healthcare utilization in COPD patients admitted with an exacerbation? A randomized controlled study. Respirology 2009; 14:230-8.

49. Holland AE, Mahal A, Hill CJ, et al. Home-based rehabilitation for COPD using minimal resources: a randomised, controlled equivalence trial. Thorax 2017;72:57-65.

50. Horton EJ, Mitchell KE, Johnson-Warrington V, et al. Comparison of a structured home-based rehabilitation programme with conventional supervised pulmonary rehabilitation: a randomised non-inferiority trial. Thorax 2018;73:29-36.

51. Johnson-Warrington V, Rees K, Gelder C, et al. Can a supported self-management program for COPD upon hospital discharge reduce readmissions? A randomized controlled trial. Int J Chron Obstruct Pulmon Dis 2016;11:1161-9.

52. Wedzicha JA, Bestall JC, Garrod R, et al. Randomized controlled trial of pulmonary rehabilitation in severe chronic obstructive pulmonary disease patients, stratified with the MRC dyspnoea scale. Eur Respir J 1998;12:363-9.

53. Jeppesen E, Brurberg KG, Vist GE, et al. Hospital at home for acute exacerbations of chronic obstructive pulmonary disease. Cochrane Database Syst Rev 2012:CD003573.

54. Murphy N, Bell C, Costello RW. Extending a home from hospital care programme for COPD exacerbations to include pulmonary rehabilitation. Respir Med 2005;99:1297-302.

55. Maddocks M, Nolan CM, Man WD, et al. Neuromuscular electrical stimulation to improve exercise capacity in patients with severe COPD: a randomised double-blind, placebo-controlled trial. Lancet Respir Med 2016;4:27-36.

56. Jones S, Man WD, Gao W, et al. Neuromuscular electrical stimulation for muscle weakness in adults with advanced disease. Cochrane Database Syst Rev 2016;10:CD009419.

57. Chaplin EJ, Houchen L, Greening NJ, et al. Neuromuscular stimulation of quadriceps in patients hospitalised during an exacerbation of COPD: a comparison of low $(35 \mathrm{~Hz})$ and high $(50 \mathrm{~Hz})$ frequencies. Physiother Res Int 2013;18:148-56.

58. Giavedoni S, Deans A, McCaughey P, et al. Neuromuscular electrical stimulation prevents muscle function deterioration in exacerbated COPD: a pilot study. Respir Med 2012;106:1429-34.

59. Zanotti E, Felicetti G, Maini M, et al. Peripheral muscle strength training in bed-bound patients with COPD receiving mechanical ventilation: effect of electrical stimulation. Chest 2003;124:292-6.

60. Cardim AB, Marinho PE, Nascimento JF Jr, et al. Does whole-body vibration improve the functional exercise capacity of subjects with COPD? A Meta-Analysis. Respir 
Care 2016;61:1552-9.

61. Greulich T, Nell C, Koepke J, et al. Benefits of whole body vibration training in patients hospitalised for COPD exacerbations - a randomized clinical trial. BMC Pulm Med 2014;14:60.

Cite this article as: Jones SE, Barker RE, Nolan CM, Patel S, Maddocks M, Man WD. Pulmonary rehabilitation in patients with an acute exacerbation of chronic obstructive pulmonary disease. J Thorac Dis 2018;10(Suppl 12):S1390-S1399. doi: $10.21037 /$ jtd.2018.03.18 